Test Method

\title{
Mechanical Characterization of Crosslinking Effect on Polydimethylsiloxane Using Nanoindentation
}

\author{
Congrui Jin ${ }^{* a}$, Zhixin Wang ${ }^{\mathrm{b}}$, Alex A. Volinsky ${ }^{\mathrm{b}}$, Asma Sharfeddin ${ }^{\mathrm{c}}$, and Nathan D. Gallant ${ }^{\mathrm{b}}$ \\ ${ }^{a}$ Department of Mechanical Engineering, State University of New York at Binghamton, Binghamton, \\ NY 13902, USA \\ ${ }^{b}$ Department of Mechanical Engineering, University of South Florida, Tampa, Florida 33620, USA \\ ${ }^{c}$ Department of Biomedical Engineering, University of South Florida, Tampa, Florida 33620, USA \\ *Corresponding author: Congrui Jin, Email: cjin@binghamton.edu
}

\begin{abstract}
Polydimethylsiloxane (PDMS) is one of the widely-used silicone-based organic polymers. It can serve as a substrate to grow cells, mainly because of its controllable range of mechanical properties. Varying the degree of crosslinking in the polymer network allows tuning its mechanical properties in a range similar to living tissues. To study the PDMS stiffness effect on the growth and behaviour of cells, it is of significant importance to explore the mechanical properties of a series of PDMS samples cured to different crosslink densities. In this paper, nanoindentation tests were conducted using spherical, Berkovich, cube corner and conical indenters to characterize PDMS mechanical properties. To interpret the nanoindentation data, the nano-JKR force curve method was successfully extended to include Berkovich, cube corner and conical indenters by conducting a numerical simulation in which the adhesive interactions are represented by an interaction potential and the surface deformations are coupled by using half-space Green's functions discretized on the surface.
\end{abstract}

Keywords: Crosslinking; Mechanical Properties; Properties and Characterization; Nanoindentation; Biomedical Applications

\section{Introduction}

Over the past decade, polydimethylsiloxane (PDMS) has been extensively employed for the fabrication of microdevices used as cell culture platforms ${ }^{1-3}$. The PDMS substrate possesses universal appeal over other materials because of its unique properties, such as biocompatibility, thermal stability, chemical inertness, optical transparency, gas permeability, lack of toxicity, low cost and easy fabrication into various shapes and sizes ${ }^{4-7}$. Most importantly, PDMS mechanical properties can be controlled by changing the weight percentage of the crosslinker, curing time and curing temperature ${ }^{8,9}$. All of these physical attributes make PDMS an ideal platform for cell-on-a-chip technology, particularly for drug discovery on microfluidic chips and microwell plates ${ }^{10-13}$. 
To investigate the PDMS stiffness effect on the growth and behaviour of cells, it is of significant importance to explore the mechanical properties of a series of PDMS samples cured to different crosslink densities. However, characterizing mechanical properties of PDMS is a challenging task, as there are multiple chemical and physical factors that can affect the mechanical properties of PDMS and limit our ability to measure these properties ${ }^{14-16}$. For example, Liu et al. demonstrated that both the mechanical strength and the Young's modulus of the PDMS membranes are thickness dependent ${ }^{14}$; Khanafer et al. found that the elastic modulus of PDMS increases with increasing strain rate ${ }^{15}$; and Wang et al. fabricated PDMS sheets with continuous stiffness gradients to study the effects of substrate stiffness on stem cell differentiation, thus rendering them non-uniform and unsuitable for macroscale tests ${ }^{16}$. While most researchers resorted to tensile testing using standard testing equipment ${ }^{3,14,15,17}$, Wang et al. utilized a custom-built macroscopic compression instrument for measuring macroscopic elastic properties of PDMS samples with 5:1 to 33:1 elastomer base to the curing agent ratios ${ }^{18}$. However, nanoindentation is capable of providing better surface sensitivity and higher spatial resolution.

Although traditional nanoindentation techniques were developed for stiff materials, such as metals and ceramics, nanoindentation testing of compliant materials, such as soft tissues, cells and hydrogels, has recently attracted substantial attention because the high spatial resolution of nanoindentation allows local testing of mechanical properties of soft matter that is not possible using macroscale techniques ${ }^{19-25}$. However, the interpretation of the measured load-displacement profiles from nanoindentation testing on soft materials has been a complex issue. One of the key concerns is the significant effect of adhesion between the indenter tip and the sample. Adhesion leads to larger contact areas and higher contact stiffness for a given applied force relative to the Hertz model ${ }^{26}$, which results in an overestimation of modulus values if the experimental data is analysed by the traditional OliverPharr analysis method implemented by most commercial software packages ${ }^{27}$. Furthermore, adhesion also makes it quite challenging to determine the initial point of contact of the indenter tip, based on which the contact area and the elastic modulus are calculated. For soft materials, because of the adhesion of the sample surface, the indenter tip is attracted to it, resulting in a negative load at the beginning of the indentation process, i.e., the so-called pull-in force. As a result, the initial contact point used for calculating the contact area and the reduced modulus in the commercial software is shifted and, therefore, the reduced modulus values automatically calculated by the software are inaccurate.

Ebenstein and Wahl sought to extract accurate modulus values from nanoindentation data by proposing a method based on the Johnson-Kendall-Roberts (JKR) model, which is now often referred to as the nano-JKR force curve method ${ }^{23,28}$. Unlike the Oliver-Pharr method, the nano-JKR force curve method seeks to fit indentation data with models that take the effect of adhesion into account. Although this approach has demonstrated its capability to correct for errors due to adhesion ${ }^{23,28}$, to our knowledge, it has not been widely adopted, mainly because it works only with perfectly spherical tips, has not been validated on a wide range of materials, and has not implemented in commercial software. In this paper, nanoindentation tests were conducted using spherical, Berkovich, cube corner and conical indenters to characterize the mechanical properties of a series of PDMS samples cured to different crosslink densities. To interpret the nanoindentation data, the nano-JKR force curve method was successfully extended to include Berkovich, cube corner and conical indenters by conducting a 
numerical simulation in which the adhesive interactions are represented by an interaction potential and the surface deformations are coupled by using half-space Green's functions discretized on the surface. The elastic modulus results of all the tested PDMS samples are compared with the results reported by a previous study using a custom-built macroscopic compression tester ${ }^{18}$.

\section{Experimental Work}

PDMS was purchased from Dow Corning Corporation as a kit of two components (Sylgard 184, Dow Corning Corporation, Midland, MI), prepolymer base and crosslinker. The components were mixed and cured to form the elastomer network. Eight different PDMS base to elastomer weight ratios were tested, 5:1, 7:1, 10:1, 16.7:1, 20:1, 25:1, 30:1 and 33:1. It was manually mixed for 15 minutes for the higher crosslinker amounts and 30 minutes for the lower crosslinker amounts. All PDMS mixtures were degassed using a vacuum pump, and then poured into clean polystyrene Petri dishes. All the samples were about 1 to $2 \mathrm{~mm}$ thick. They were cured at $65^{\circ} \mathrm{C}$ for 20 to 24 hours. To accommodate the nanoindentation workspace, the prepared PDMS sheets were cut into $1 \mathrm{~cm}^{2}$ square pads with a utility knife. Instead of the typical glue mounting of the sample, it was placed directly onto the Hysitron Triboindenter stage and pressed with tweezers to develop full contact with the stage, evident by the air escaping along the interface between the sample and the steel sample stage holder.

Indentation was performed using a Hysitron TI-950 TriboIndenter (Hysitron, Inc., Minneapolis, MN) equipped with commercially available diamond tips (Hysitron, Inc., Minneapolis, MN). Four different tip geometries were used for testing: a $74.4 \mu \mathrm{m}$ radius spherical tip, a Berkovich tip, a cube corner tip, and a conical tip with $90^{\circ}$ conical angle. All indents captured full force curves in displacement control. The indenter first detected the surface with a setpoint force of $2 \mu \mathrm{N}$, then lifted off from the surface at a rate of $-150 \mathrm{~nm} / \mathrm{s}$ to a height of $1800 \mathrm{~nm}$ above the surface, well outside the adhesive interaction zone, and finally indented the sample at a constant displacement rate of $60 \mathrm{~nm} / \mathrm{s}$ to the maximum depth. At least five tests were conducted for each combination of indenter type and PDMS stiffness.

\section{Numerical Simulation}

Since no analytical solution exists for the force-displacement relationship for Berkovich, cube corner, or conical indenters, in this section, numerical simulations will be conducted based on the governing equations of adhesive contact problems involving non-spherical shapes. For surface atomic interaction, the empirical potential we used here is the Lennard-Jones potential ${ }^{29}$. Hence, we can obtain the relationship between the local pressure $p$ and the air gap $h$ as follows ${ }^{30}$ :

$$
p(h)=\frac{8 W_{a d}}{3 \varepsilon}\left[(\varepsilon / h)^{9}-(\varepsilon / h)^{3}\right]
$$

where $W_{a d}$ is the work of adhesion, the tensile force integrated over the distance necessary to pull apart the two bodies, and $\varepsilon$ is a length parameter equal to the range of the surface interaction. Derjaguin's approximation $^{31}$ is then applied to Eqn. (1). The separation between the two surfaces due to the surface interaction as well as the applied load, denoted by $h$, will be expressed by the following equation ${ }^{31}$ : 


$$
h(x, y)=-\alpha+\varepsilon+h_{0}+\frac{1}{\pi E^{*}} \iint_{\Omega} \frac{p\left(x^{\prime}, y^{\prime}\right) d x^{\prime} d y^{\prime}}{\sqrt{\left(x-x^{\prime}\right)^{2}+\left(y-y^{\prime}\right)^{2}}}
$$

where the parameter $\alpha$ is the displacement between the two surfaces with respect to the zero force position $h=\varepsilon$, i.e., the so called indentation depth, and $E^{*}$ represents the effective elastic modulus, also termed the reduced modulus. For two linearly elastic isotropic materials with Young's modulus $E_{i}$ and Poisson's ratio $v_{i}$, where $i=1,2, E^{*}$ is defined as follows ${ }^{31}$ :

$$
\frac{1}{E^{*}}=\frac{1-v_{1}^{2}}{E_{1}}+\frac{1-v_{2}^{2}}{E_{2}}
$$

Note that, in our simulations, Derjaguin's approximation is used to calculate surface traction, which is not the exact adhesion. If the Derjaguin assumptions are not accepted and/or there is friction at the edge of the contact region, then the adhesive forces can work on tangential displacements and the mode-mixity effects have to be discussed. As Borodich et al. noted ${ }^{32}$, interaction between adhesion and friction under both static and kinetic conditions is still an open question.

The parameter $h_{o}$ is the separation of the two surfaces in the absence of applied and adhesive forces. For spherical indenters, in the JKR model, the geometry of a spherical contact is approximated by a parabolic function $^{31}$ :

$$
h_{0}(x, y)=\frac{x^{2}+y^{2}}{2 R}
$$

where $R$ is the radius of the sphere indenter. For Berkovich and cube corner indenters, the geometry of the tip is a pyramid having a triangular base ${ }^{33}$. The initial air gap for a pyramid with a base of an equilateral triangle with side length $m$ and a height of $n$ can be written as follows:

$h_{0}(x, y)=\left\{\begin{array}{l}\frac{n}{m}(\sqrt{3} y+3|x|) \text { if }|x|>-\sqrt{3} y \\ \frac{2 \sqrt{3} n|y|}{m} \text { otherwise }\end{array}\right.$

For a Berkovich tip, the angle between the centerline and the three faces is $65.3^{\circ}$, which gives $n / m=0.133$. However, in the case of a cube corner indenter, the centerline-to-face angle is $35.3^{\circ}$, much sharper than a Berkovich tip, and we have $n / m=0.408$. For conical indenters, the initial air gap for an indenter of a right cone with height $h$ and base radius $R$ can be written as the following:

$$
h_{0}(x, y)=\frac{h\left(x^{2}+y^{2}\right)^{1 / 2}}{R}
$$

The total normal load $f$ can be written as follows:

$$
f=\iint_{\Omega} p(x, y) d x d y
$$

We then introduce the following dimensionless variables: $H, D, U_{0}, P, X, Y, F$, and $\mu$ to transform Eqns. (1), (2) and (7) into the following normalized forms:

$$
\begin{aligned}
& P=\frac{8}{3}\left[(H+1)^{-9}-(H+1)^{-3}\right] \\
& H=-D+U_{0}+\frac{8 \mu^{3 / 2}}{3 \pi} \iint_{\Omega} \frac{\left[H\left(X^{\prime}, Y^{\prime}\right)+1\right]^{-9}-\left[H\left(X^{\prime}, Y^{\prime}\right)+1\right]^{-3}}{\sqrt{\left(X-X^{\prime}\right)^{2}+\left(Y-Y^{\prime}\right)^{2}}} d X^{\prime} d Y^{\prime}
\end{aligned}
$$


$F=\frac{1}{3 \pi} \iint_{\Omega} P(X, Y) d X d Y$

where $H=\frac{h}{\varepsilon}-1, D=\frac{\alpha}{\varepsilon}, U_{0}=\frac{h_{0}}{\varepsilon}$, and $P=\frac{p \varepsilon}{W_{a}}$. The parameter $D$ is the normalized indentation depth. For a spherical shape, we have $F=\frac{f}{3 \pi R W_{\alpha}}, X=\frac{x}{\sqrt{\varepsilon R}}, Y=\frac{y}{\sqrt{\varepsilon R}}$, and $\mu=\left(\frac{R W_{\alpha}^{2}}{E^{2 / 2} \varepsilon^{3}}\right)^{1 / 3}$, and the initial air gap can be written as:

$U_{0}(x, y)=\frac{X^{2}+Y^{2}}{2}$

For a Berkovich shape or a cube corner shape, we have $F=\frac{n^{2} f}{3 \pi m^{2} W_{\alpha \phi^{2}}}, X=\frac{n x}{\varepsilon m}, Y=\frac{n y}{\varepsilon m}$, and $\mu=\left(\frac{m W_{\alpha \mu}}{n E^{*} \varepsilon}\right)^{2 / 3}$.

The initial air gap can be described as:

$U_{0}(x, y)=\left\{\begin{array}{l}\sqrt{3} Y+3|X| \text { if }|X|>-\sqrt{3} Y \\ 2 \sqrt{3}|Y| \text { otherwise }\end{array}\right.$

For a conical shape, we have $F=\frac{h^{2} f}{3 \pi R^{2} W_{\omega} \varepsilon}, X=\frac{h x}{R \varepsilon}, Y=\frac{h y}{R \varepsilon}$, and $\mu=\left(\frac{R W_{\alpha \mu}}{h E^{*} \varepsilon}\right)^{2 / 3}$, and the initial air gap can be written as: $U_{0}(x, y)=\left(X^{2}+Y^{2}\right)^{1 / 2}$

Note that the parameter $\mu$ is the so called Tabor parameter ${ }^{34}$, which is often used to determine whether the JKR or DMT model would best describe a contact system. Eqn. (9) is then solved by a virtual state relaxation (VSR) method: the indentation depth $D$ is gradually increased, and the $H$ vector obtained from the previous step is used as an initial state for computing $H$ vector in the next step. In each step, we let time evolve until the final state in equilibrium is reached. This method accurately plots all the stable equilibria for each value of $D$. In all the numerical simulations, we first increase the value of $D$ from minimum to the maximum indentation depth to simulate the approach process, and then we decrease the value of $D$ back to the minimum to simulate the detachment process. This method has been extensively calibrated and validated in recent years, showing superior capability in reproducing and predicting the contact behavior of adhesive materials in various kinds of contact problems $^{35-38}$.

\section{Results and Discussion}

\subsection{Spherical Indenter}

We first simulated the nanoindentation using spherical indenters because, for this case, the analytical solution is known ${ }^{26}$ and, consequently, provides a baseline to compare against. For the spherical shape, we found that when the value of Tabor parameter is 1.5 , the numerical results agree very well with the analytical solution derived from the JKR model, as shown in Fig. 1(a), which plots the numerical 
curves of the normalized force $F=\frac{f}{3 \pi R W_{\alpha}}$ versus the normalized displacement $D=\frac{\alpha}{\varepsilon}$ for both attachment and detachment processes. It can be seen that the numerical simulation results successfully capture the pull-in and the pull-off behavior often observed in nanoindentation experiments ${ }^{39}$. Fig. 1(a) also plots the distribution of the normalized pressure $\mathrm{P}$ in the simulation domain $[-4.0,4.0] \times[-4.0,4.0]$ when the normalized indentation depths are $\mathrm{D}=-2.5,-1.8$, and -1.0 during approach. It can be seen that when the two bodies are approaching each other from a noncontact state, e.g. at $\mathrm{D}=-2.5$, the surfaces barely deform with pressure being nearly zero everywhere. As the two bodies approach each other one step further, e.g., at $\mathrm{D}=-1.8$, the surfaces jump to a new equilibrium state suddenly with a nonzero contact area, and the pressure becomes compressive in the central region and tensile at the contact edge, which is consistent with the results from previous studies ${ }^{31}$.

A representative loading and unloading force-displacement curve obtained from 20:1 PDMS using the $74.4 \mu \mathrm{m}$ radius spherical tip is shown in Fig. 1(b). The observed adhesion hysteresis shows that the work of adhesion during approach, denoted as $W_{a d}^{a p}$, is much lower than that during detachment, denoted as $W_{a d}^{d e}$. According to the nano-JKR force curve method ${ }^{23}$, the values of $W_{a d}^{a p}$ and $W_{a d}{ }^{d e}$ can be calculated from the measured pull-off force $f_{o f f}^{a p}$ during approach and $f_{\text {off }}^{\text {de }}$ during detachment by using the relationship: $W_{a d}^{a p}=\frac{2}{3} \frac{f_{o f f}^{a p}}{\pi R}$ and $W_{a d}^{d e}=\frac{2}{3} \frac{f_{o f}^{d e}}{\pi R}$, respectively. $E^{*}$ can be calculated based on two data points from the unloading portion of the curve, $f_{o f}^{d e}$ at $\alpha_{o f f}^{d e}$ (the point where the unloading curve reaches its minimum force) and $f_{\text {zеro }}^{d e}$ at $\alpha_{\text {zero }}{ }^{d e}$ (the point where the unloading force equals zero), and the relationship obtained from the JKR model: $E^{*}=\frac{0.95 f_{o f f}^{d e}}{\sqrt{R}}\left(\alpha_{z e r o}^{d e}-\alpha_{o f f}^{d e}\right)^{-3 / 2}$. Since we can measure that $f_{o f}^{d e}=29.46 \mu N, f_{o f f}^{a p}=6.57 \mu N$, and $\alpha_{z e r o r}^{d e}-\alpha_{o f}^{d e}=2040 \mathrm{~nm}$, substituting these values into the nano-JKR force curve equations, we can obtain that $W_{a d}^{a p}=0.019 \mathrm{Jm}^{-2}, W_{a d}^{d e}=0.084 \mathrm{Jm}^{-2}$, and $E^{*}=1.11 \mathrm{MPa}$, all of which are reasonable values according to our previous experimental experience ${ }^{36,40}$. Fig. 1(b) shows the force-displacement curve obtained from numerical simulations in a dimensional scale superimposed with the experimental data. The mechanical properties of the PDMS samples with other values of stiffness were characterized in a similar manner.

\subsection{Berkovich Indenter}

For the Berkovich shape and the cube corner shape, Fig. 2(a) plots the numerical simulation curves of the normalized force $F=\frac{n^{2} f}{3 \pi m^{2} W_{\alpha} \varepsilon}$ versus the normalized displacement $D=\frac{\alpha}{\varepsilon}$ for both attachment and detachment processes when the value of the Tabor parameter is equal to 1.5. Based on the numerical simulation result, we can see that the normalized pull-off force $F_{o f f}=0.21$, the unloading force reaches its 
minimum at $D_{o f f}^{d e}=1.59$, and when the unloading force equals zero, $D_{z e r o}^{d e}=5.31$. These values will be used to calculate $W_{a d}^{a p}, W_{a d}^{d e}$, and $E^{*}$. The pull-in behavior is not as obvious, because for the Berkovich and cube corner indenters, the contact area at a given amount of indentation depth is much smaller than that obtained from the nanoidentation tests using spherical indenters. Fig. 2(a) also plots the distribution of the normalized pressure $\mathrm{P}$ in the simulation domain $[-6.7,6.7] \times[-6.7,6.7]$ when the normalized indentation depths are $\mathrm{D}=-2.5,2.5$, and 5.5 during approach.

Since we have $F_{\text {off }}=0.21$, the relationship of $W_{a d}^{a p}$ and $W_{a d}^{d e}$ with the measured pull-off force $f_{o f f}^{a p}$ during approach and $f_{o f f}^{d e}$ during detachment can be written as: $W_{a d}^{a p}=\frac{n^{2} f_{o f}^{a p p}}{0.63 \pi m^{2} \varepsilon}$ and $W_{a d}^{d e}=\frac{n^{2} f_{o f f}^{d e}}{0.63 \pi m^{2} \varepsilon}$, respectively. From $\mu=\left(\frac{m W_{a d}}{n E^{*} \varepsilon}\right)^{2 / 3}=1.5$, we can obtain that $\varepsilon=\frac{m W_{a d}}{1.84 n E^{*}}$. Since $W_{a d}^{a p}$ is lower than $W_{a d}^{d e}, \varepsilon^{a p}=\frac{m W_{a d}^{a p}}{1.84 n E^{*}}$ should be smaller than $\varepsilon^{d e}=\frac{m W_{a d}^{d e}}{1.84 n E^{*}}$. We then have the following equations, respectively:

$W_{a d}^{a p}=\left(\frac{2.92 n^{3} f_{o f f}^{a p} E^{*}}{\pi m^{3}}\right)^{1 / 2}$
$W_{a d}^{d e}=\left(\frac{2.92 n^{3} f_{o f}^{d e} E^{*}}{\pi m^{3}}\right)^{1 / 2}$

Since the numerical simulation curve shows that $D_{z e r o}^{d e}-D_{o f f}^{d e}=3.72$, we have $\alpha_{z e r o}^{d e}-\alpha_{o f}^{d e}=3.72 \varepsilon$, and then we can obtain the following relationship:

$\alpha_{z e r o}^{d e}-\alpha_{o f f}^{d e}=\frac{2.02 m W_{a d}^{d e}}{n E^{*}}$

Eqn. (14), Eqn. (15), and Eqn. (16) can be used to determine $W_{a d}^{a p}, W_{a d}{ }^{d e}$, and $E^{*}$ for a compliant material based on the nanoindentation tests using Berkovich indenters and cube corner indenters. Note that for the Berkovich indenter, we have $\mathrm{n} / \mathrm{m}=0.133$; whereas for the cube corner indenter, we have $\mathrm{n} / \mathrm{m}=0.408$.

A complete loading and unloading force-displacement curve obtained from 16.7:1 PDMS using the Berkovich tip is shown in Fig. 2(b), from which we can obtain that $f_{o f f}^{d e}=3.39 \mu N, f_{o f f}^{a p}=0.77 \mu N$, and $\alpha_{z e r o}^{d e}-\alpha_{o f f}^{d e}=1166 \mathrm{~nm}$. Substituting these values into Eqns. (14), (15), and (16), we can obtain that $W_{a d}^{a p}=0.046 \mathrm{Jm}^{-2}, W_{a d}^{d e}=0.097 \mathrm{Jm}^{-2}$, and $E^{*}=1.26 \mathrm{MPa}$. The result showing that the reduced modulus slightly increased from 1.11 MPa for 20:1 PDMS to 1.26 MPa for 16.7 PDMS is consistent with our expectations, since the higher the degree of PDMS network's crosslinking, the stiffer the sample will be ${ }^{18,40}$. Fig. 2(b) also shows the force-displacement curve obtained from numerical simulations in a dimensional scale based on $W_{a d}^{a p}=0.046 \mathrm{Jm}^{-2}, W_{a d}{ }^{d e}=0.097 \mathrm{Jm}^{-2}$, and $E^{*}=1.26 \mathrm{MPa}$ superimposed with the experimental data. 


\subsection{Cube Corner Indenter}

To analyze the results from the cube corner indenters, a complete loading and unloading forcedisplacement curve obtained from 5:1 PDMS is shown in Fig. 3(a), from which we can obtain that $f_{o f}^{d e}=1.90 \mu \mathrm{N}, f_{o f}^{a p}=0.57 \mu \mathrm{N}$, and $\alpha_{z w o}^{d e}-\alpha_{o f}^{d e}=902 \mathrm{~nm}$. Substituting these values into Eqns. (14), (15), and (16) and keeping in mind that, for the cube corner indenter, we have $\mathrm{n} / \mathrm{m}=0.408$, we can obtain that $W_{a d}^{a p}=0.361 \mathrm{Jm}^{-2}, W_{a d}^{d e}=0.659 \mathrm{Jm}^{-2}$, and $E^{*}=3.63 \mathrm{MPa}$. Fig. 3(a) shows the force-displacement curve obtained from numerical simulations in a dimensional scale based on $W_{a d}^{a p}=0.361 \mathrm{Jm}^{-2}, W_{a d}^{d e}=0.659 \mathrm{Jm}^{-2}$, and $E^{*}=3.63 \mathrm{MPa}$ superimposed with the experimental data. The result of $E^{*}=3.63 \mathrm{MPa}$ for 5:1 PDMS is in good agreement with the experimental results obtained from the macroscopic compression test ${ }^{18}$. To check if it is consistent with the results obtained from the nanoindentation tests using other types of indenters, a complete loading and unloading force-displacement curve obtained from 5:1 PDMS using the Berkovich indenter is in Fig. 3(b), from which we can obtain $f_{o f}^{d e}=1.13 \mu N, f_{o f}^{a p}=0.90 \mu N$, and $\alpha_{z x o}^{u^{k}}-\alpha_{o f}^{d e}=389 \mathrm{~nm}$. Substituting these values in Eqns. (14), (15), and (16) and using $\mathrm{n} / \mathrm{m}=0.133$, we can obtain $W_{\text {ad }}^{\text {ap }}=0.086 \mathrm{Jm}^{-2}, W_{\text {ad }}^{d e}=0.097 \mathrm{Jm}^{-2}$, and $E^{*}=3.78 \mathrm{MPa}$. The value of $E^{*}$ is slightly higher but fairly consistent with the result obtained from the nanoindentation tests using the cube corner indenter. Fig. 3(b) shows the force-displacement curve obtained from numerical simulations in a dimensional scale based on $W_{a d}^{a p}=0.086 \mathrm{Jm}^{-2}, W_{a d}^{d e}=0.097 \mathrm{Jm}^{-2}$, and $E^{*}=3.78 \mathrm{MPa}$ superimposed with the experimental data.

\subsection{Conical Indenter}

For the conical indenter, Fig. 4(a) plots the numerical simulation curves of the normalized force $F=\frac{h^{2} f}{3 \pi R^{2} W_{\alpha^{\prime}}}$ versus the normalized displacement $D=\frac{\alpha}{\varepsilon}$ for both attachment and detachment processes when the value of the Tabor parameter is equal to 1.5. Based on the numerical simulation result, we can see that the normalized pull-off force $F_{\text {off }}=3.57$, the unloading force reaches its minimum at $D_{o f}^{d e}=5.46$, and when the unloading force equals zero, $D_{z r o}^{d_{c}}=15.00$. These values will be used to calculate $W_{a d}^{a p}, W_{a d}{ }^{d e}$, and $E^{*}$. Fig. 4(a) also plots the distribution of the normalized pressure $\mathrm{P}$ in the simulation domain $[-4.0,4.0] \times[-4.0,4.0]$ when the normalized indentation depths are $\mathrm{D}=-3.0,-1.0$, and -0.1 during approach. It can be seen that, for conical indenters, the maximum compressive pressure at the center of the contact area forms a sharp peak.

Since we have $F_{\text {off }}=3.57$, the relationship of $W_{a d}^{a p}$ and $W_{a d}{ }^{d e}$ with the measured pull-off force $f_{o f}$ ap during approach and $f_{o f}^{d e}$ during detachment can be written as: $W_{a d}^{a p}=\frac{h^{2} f_{o f}^{a p}}{10.71 \pi R^{2} \varepsilon}$ and $W_{a d}^{d e}=\frac{h^{2} f_{o f}^{d e}}{10.71 \pi R^{2} \varepsilon}$, 
respectively. From $\mu=\left(\frac{R W_{a d}}{h E^{*} \varepsilon}\right)^{2 / 3}=1.5$, we can obtain that $\varepsilon=\frac{R W_{a d}}{1.84 h E^{*}}$. Since $W_{a d}^{a p}$ is lower than $W_{a d}^{d e}$, $\varepsilon^{a p}=\frac{R W_{a d}^{a p}}{1.84 h E^{*}}$ should be smaller than $\varepsilon^{d e}=\frac{R W_{a d}^{d e}}{1.84 h E^{*}}$. We then have the following equations, respectively:

$W_{a d}^{a p}=\left(\frac{h^{3} f_{o f f}^{a p} E^{*}}{5.82 \pi R^{3}}\right)^{1 / 2}$

$W_{a d}^{d e}=\left(\frac{h^{3} f_{o f f}^{d e} E^{*}}{5.82 \pi R^{3}}\right)^{1 / 2}$

Since the numerical simulation curve shows that $D_{z e r o}^{d e}-D_{o f f}^{d e}=9.54$, we have $\alpha_{z e r o}^{d e}-\alpha_{o f f}^{d e}=9.54 \varepsilon$, and then we can obtain the following relationship:

$\alpha_{z e r o}^{d e}-\alpha_{o f f}^{d e}=\frac{5.18 R W_{a d}^{d e}}{h E^{*}}$

Eqn. (17), Eqn. (18), and Eqn. (19) can be used to determine $W_{a d}^{a p}, W_{a d}^{d e}$, and $E^{*}$ for a compliant material based on the nanoindentation tests using conical indenters. The ratio of $\mathrm{R} / \mathrm{h}$ can be obtained from the value of the conical angle. For the conical indenter with $90^{\circ}$ conical angle, we have $\mathrm{R} / \mathrm{h}=1$.

A complete loading and unloading force-displacement curve obtained from 5:1 PDMS using a conical tip with $90^{\circ}$ conical angle has been shown in Fig. 4(b), from which we can obtain that $f_{\text {off }}^{d e}=4.15$ $\mu N, f_{o f f}^{a p}=1.20 \mu N$, and $\alpha_{z e r o}^{d e}-\alpha_{o f f}^{d e}=1330 \mathrm{~nm}$. Substituting these values and the value of $\mathrm{R} / \mathrm{h}=1$ into Eqns. (17), (18), and (19), we can obtain that $W_{a d}^{a p}=0.475 \mathrm{Jm}^{-2}, W_{a d}^{d e}=0.884 \mathrm{Jm}^{-2}$, and $E^{*}=3.44 \mathrm{MPa}$. Fig. 4(b) also shows the force-displacement curve obtained from numerical simulations in a dimensional scale based on $W_{a d}^{a p}=0.475 \mathrm{Jm}^{-2}, W_{a d}{ }^{d e}=0.884 \mathrm{Jm}^{-2}$, and $E^{*}=3.44 \mathrm{MPa}$ superimposed with the experimental data.

The value of $E^{*}$ is slightly lower than the values obtained by using the Berkovich indenter and the cube corner indenter. Several factors, different in nature, could explain the discrepancy in the modulus results. First, slight variations from the ideal geometries used in the numerical simulations would be expected for the real tips due to blunted points in the three tips. Second, in numerical simulations, Derjaguin's approximation is used to calculate surface traction, which is not the exact adhesion. Finally, the formulas used in the numerical simulation are restricted in the small strain range and linear elastic material behavior. Those assumptions can lead to some error in modulus calculation.

\subsection{Crosslinking Effect}

Since the elastic modulus of the diamond indenter tip is orders of magnitude larger than the elastic modulus of PDMS, the second term in Eqn. (3) is negligible. Hence, using the reduced modulus of PDMS, $E^{*}$, and the Poisson's ratio, $v$, the elastic modulus of PDMS, E, can be calculated as $E=\left(1-v^{2}\right) E^{*}=0.75 E^{*}$. The elastic modulus results of the tested PDMS samples versus the PDMS 
base/curing agent weight ratio, $n$, are listed in Table 1 . It is clear that the PDMS elastic modulus is related to the elastomer base/curing agent ratio, i.e., the degree of crosslinking. The elastic modulus, $E$, in MPa can be expressed as a function of the PDMS base/curing agent weight ratio, $n$, as $E=\frac{20 M P a}{n}$, as shown in Fig. 5. In a previous study, Wang et al. utilized a custom-built macroscopic compression tester to measure the elastic modulus of a series of PDMS samples with different crosslink densities ${ }^{18}$, and the results were also plotted in Fig. 5 for the purpose of comparison. It can be seen that the elastic modulus results of the tested PDMS samples obtained from nanoindentation tests show good agreement with the results obtained from the compression tests.

\section{Conclusions}

Although the mechanical properties of PDMS have been previously studied, none of these investigations focused on the change of the elastic modulus over a wide range of the crosslinking weight $\operatorname{ratios}^{41-43}$. Most of the studies only tested two or three different stiffnesses, which is not enough to fully understand the crosslinking effect on the PDMS elastic modulus. In this study, we have conducted nanoindentation tests using different types of indenters to characterize the mechanical properties of PDMS with a much wider range of crosslinking ratios. To interpret the measured loaddisplacement profiles from nanoindentation tests, we have successfully extended the nano-JKR force curve method to include Berkovich, cube corner, and conical indenters by conducting a numerical simulation in which the adhesive interactions are represented by an interaction potential and the surface deformations are coupled by using half-space Green's functions discretized on the surface. The elastic modulus results of the tested PDMS samples show good agreement with the results reported by a previous study using a custom-built macroscopic compression tester. The numerical simulation package can be easily extended to study nanoindentation tests using other types of indenter, such as Vickers indenters ${ }^{44}$, Knoop indenters ${ }^{45}$, cylindrical flat indenters and frustum indenters, which will be of significant importance for mechanical characterization of polymers and other compliant materials.

\section{Acknowledgement}

This work is supported by start-up funds provided by the Department of Mechanical Engineering at State University of New York at Binghamton.

\section{Notes and references}

1. W. Zhang, D. S. Choi, Y. H. Nguyen, J. Chang, L. Qin, Sci. Rep. 2013, 3, 2332.

2. X. Q. Brown, K. Ookawa, J. Y. Wong, Biomaterials 2005, 26, 3123-9.

3. N. Eroshenko, R. Ramachandran, V. K. Yadavalli, R. R. Rao, J. Biol. Eng. 2013, 7, 7.

4. J. C. McDonald, G. M. Whitesides, Acc. Chem. Res. 2002, 35, 491-499.

5. S. K. Sia, G. M. Whitesides, Electrophoresis 2003, 24, 3563-3576.

6. D. A. Tolle, C. L. Frye, R. G. Lehmann, T. C. Zwick, Sci. Total Environ. 1995, 162, 193-207. 
7. Y. Zhao, C. C. Lim, D. B. Sawyer, R. Liao, X. Zhang, J. Micromech. Microeng. 2005, 15, 9.

8. A. Mata, A. J. Fleischman, S. Roy, Biomed. Microdevices 2005, 7, 281.

9. G. Bartalena, Y. Loosli, T. Zambelli, J. Snedeker, Soft Matter 2012, 8, 673.

10. J. El-Ali, P. K. Sorger, K. F. Jensen, Cells on chips. Nature 2006, 442, 403-411.

11. P. S. Dittrich, A. Manz, Nat. Rev. Drug Discov. 2006, 5, 210-218.

12. J. C. Love, J. L. Ronan, G. M. Grotenbreg, A. G. van der Veen, H. L. Ploegh, Nat. Biotechnol. 2006, 24, 703-707.

13. B. Cortese, G. Gigli, M. Riehle, Adv. Funct. Mater. 2009, 19, 2961.

14. M. Liu, J. Sun, Y. Sun, C. Bock, Q. Chen, J. Micromech. Microeng. 2009, 19, 035028.

15. K. Khanafer, A. Duprey, M. Schlicht, R. Berguer, Biomed. Microdevices 2009, 11, 503.

16. P. Wang, W. Tsai, N. H. Voelcker, Acta Biomater. 2012, 8, 519.

17. R. N. Palchesko, L. Zhang, Y. Sun, A. W. Feinberg, PLoS One 2012, 7, e51499.

18. Z. Wang, A. A. Volinsky, N. D. Gallant, J. Appl. Polym. Sci. 2014, 131, 41050.

19. K. J. Tong, D. M. Ebenstein, JOM 2015, 67, 713.

20. J. C. Kohn, D. M. Ebenstein, Journal of the mechanical behavior of biomedical materials 2013, 20, 316.

21. V. L. Ferguson, A. J. Bushby, A. Boyde, Journal of Anatomy 2003, 203, 191.

22. P. L. Leong, E. F. Morgan, Acta Biomaterialia 2008, 4, 1569.

23. D. M. Ebenstein, J. Mater. Res. 2011, 28, 1026.

24. F. Alisafaei, C.-S. Han, S. H. R. Sanei, Polym. Test. 2013, 32, 1220.

25. C. Klapperich, L. Pruitt, K. Komvopoulos, J. Mater. Res. 2002, 17, 423.

26. K. L. Johnson, Contact Mechanics, Cambridge University Press, Cambridge, 1985.

27. W. C. Oliver, G. M. Pharr, J. Mater. Res. 1992, 7, 1564.

28. D. M. Ebenstein, K. J. Wahl, J. Colloid Interface Sci. 2006, 298, 652.

29. J. N. Israelachvili, Intermolecular and Surface Forces, 2nd ed. Academic, San Diego, 1992.

30. N. Yu, A. Polycarpou, J. Colloid Interface Sci. 2004, 278, 428.

31. J. A. Greenwood, Proc. R. Soc. Lond. A 1997, 453, 1277.

32. F. M. Borodich, B. A. Galanov, M. M. Suarez-Alvarez, J. Mech. Phys. Solids 2014, 68, 14.

33. E. S. Berkovich, Int. Diamond Rev. 1951, 11, 129.

34. D. Tabor, J. Colloid Interface Sci. 1977, 58, 2.

35. C. Jin, J. Adhes. Sci. Technol. 2016, 30, 1223-1242.

36. C. Jin, K. Khare, S. Vajpayee, S. Yang, A. Jagota, C.-Y. Hui, Soft Matter 2011, 7, 10728.

37. C. Jin, A. Jagota, C.-Y. Hui, J. Phys. D. Appl. Phys. 2011, 44, 405303.

38. C. Jin, D. S. Kia, M. Jones, S. Towfighian, Nano Energy 2016, 27, 68-77.

39. Z. Wang, A. A. Volinsky, N. D. Gallant, J. Appl. Polym. Sci. 2015, 132, 41384.

40. A. Sharfeddin, A. A. Volinsky, G. Mohan, N. D. Gallant, J. Appl. Polym. Sci. 2015, 132, 42680.

41. S. Gupta, F. Carrillo, C. Li, L. Pruitt, C. Puttlitz, Mater. Lett. 2007, 61, 448.

42. F. Carrillo, S. Gupta, M. Balooch, S. J. Marshall, G. W. Marshall, L. Pruitt, C. M. Puttlitz, J. Mater.

Res. 2005, 20, 2820.

43. D. S. Gray, J. Tien, C. S. Chen, J. Biomed. Mater. Res. Part A 2003, 66, 605.

44. R. L. Smith, G. E. Sutherland, Iron Steel Inst. 1925, 1, 285.

45. F. Knoop, C. G. Peters, W. B. Emerson, J. Res. Nat. Bur. Stand. 1939, 23, 39. 


\section{Graphical Abstract}

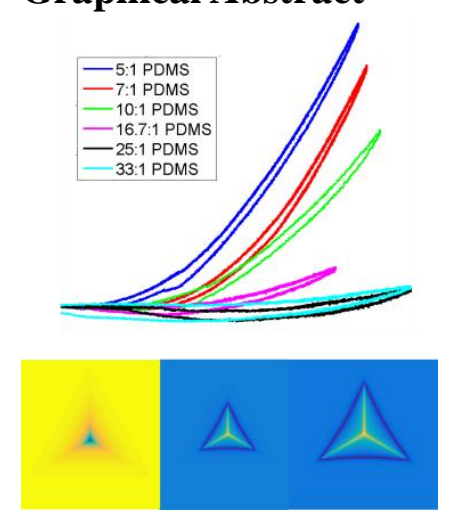

Table 1: Elastic Modulus of PDMS Network Samples with Different Amount of Crosslinking

\begin{tabular}{|c|c|c|c|c|c|}
\hline Base to Crosslinker Ratio, n $5: 1 \quad 7: 1 \quad 10: 1$ & $16.7: 1$ & $20: 1$ & $25: 1$ & $30: 1$ & $33: 1$ \\
\hline $\begin{array}{llll}\mathrm{E}(\mathrm{MPa}) & 3.62 \pm 0.19 & 2.91 \pm 0.17 & 1.75 \pm 0.18\end{array}$ & $1.26 \pm 0.19$ & $1.11 \pm 0.20$ & $0.98 \pm 0.09$ & $0.80 \pm 0.09$ & $0.56 \pm 0.04$ \\
\hline
\end{tabular}

\section{Figure Captions}

Fig. 1 (a) The numerical curves of the normalized force F versus the normalized displacement D for the spherical indenter for both attachment and detachment processes. Also shown is the distribution of the normalized pressure $\mathrm{P}$ in the simulation domain $[-4.0,4.0] \times[-4.0,4.0]$ when the normalized indentation depth $\mathrm{D}=-2.5,-1.8$, and -1.0 during approach. (b) The force-displacement curve in a dimensional scale based on $W_{a d}^{a p}=0.019 \mathrm{Jm}^{-2}, W_{a d}{ }^{d e}=0.084 \mathrm{Jm}^{-2}$, and $E^{*}=1.11 \mathrm{MPa}$ superimposed with the experimental data from nanoindentation tests on 20:1 PDMS using the $74.4 \mu m$ radius spherical indenter.

Fig. 2 (a) The numerical curves of the normalized force F versus the normalized displacement D for the Berkovich indenter for both attachment and detachment processes. The value of Tabor parameter is set to be 1.5. Also shown is the distribution of the normalized pressure $\mathrm{P}$ in the simulation domain $[-6.7,6.7] \times[-6.7,6.7]$ when the normalized indentation depth $\mathrm{D}=-2.5,2.5$, and 5.5 during approach. (b) The force-displacement curve in a dimensional scale based on $W_{a d}^{a p}=0.046 \mathrm{Jm}^{-2}, W_{a d}^{d e}=0.097 \mathrm{Jm}^{-2}$, and $E^{*}=1.26 \mathrm{MPa}$ superimposed with the experimental data from nanoindentation tests on 16.7:1 PDMS using the Berkovich indenter.

Fig. 3 (a) The force-displacement curve in a dimensional scale based on $W_{a d}^{a p}=0.361 \mathrm{Jm}^{-2}, W_{a d}^{d e}=0.659$ $\mathrm{Jm}^{-2}$, and $E^{*}=3.63 \mathrm{MPa}$ superimposed with the experimental data from nanoindentation tests on 5:1 PDMS using the cube corner indenter. (b) The force-displacement curve in a dimensional scale based 
on $W_{a d}^{a p}=0.086 \mathrm{Jm}^{-2}, W_{a d}{ }^{d e}=0.097 \mathrm{Jm}^{-2}$, and $E^{*}=3.78 \mathrm{MPa}$ superimposed with the experimental data from nanoindentation tests on 5:1 PDMS using the Berkovich indenter.

Fig. 4 (a) The numerical curves of the normalized force F versus the normalized displacement D for the conical indenter with $90^{\circ}$ conical angle for both attachment and detachment processes. The value of Tabor parameter is set to be 1.5. Also shown is the distribution of the normalized pressure $\mathrm{P}$ in the simulation domain $[-4.0,4.0] \times[-4.0,4.0]$ when the normalized indentation depths are $\mathrm{D}=-3.0,-1.0$, and 0.1 during approach. (b) The force-displacement curve in a dimensional scale based on $W_{a d}^{a p}=0.475 \mathrm{Jm}^{-2}$, $W_{a d}^{d e}=0.884 \mathrm{Jm}^{-2}$, and $E^{*}=3.44 \mathrm{MPa}$ superimposed with the experimental data from nanoindentation tests on 5:1 PDMS using the conical indenter.

Fig. 5 The elastic modulus, $E$, in MPa can be expressed as a function of the PDMS base/curing agent weight ratio, $n$, as $E=\frac{20 M P a}{n}$. It also shows that the elastic modulus results of the tested PDMS samples obtained from nanoindentation tests show good agreement with the results obtained from the compression tests. 
(a)

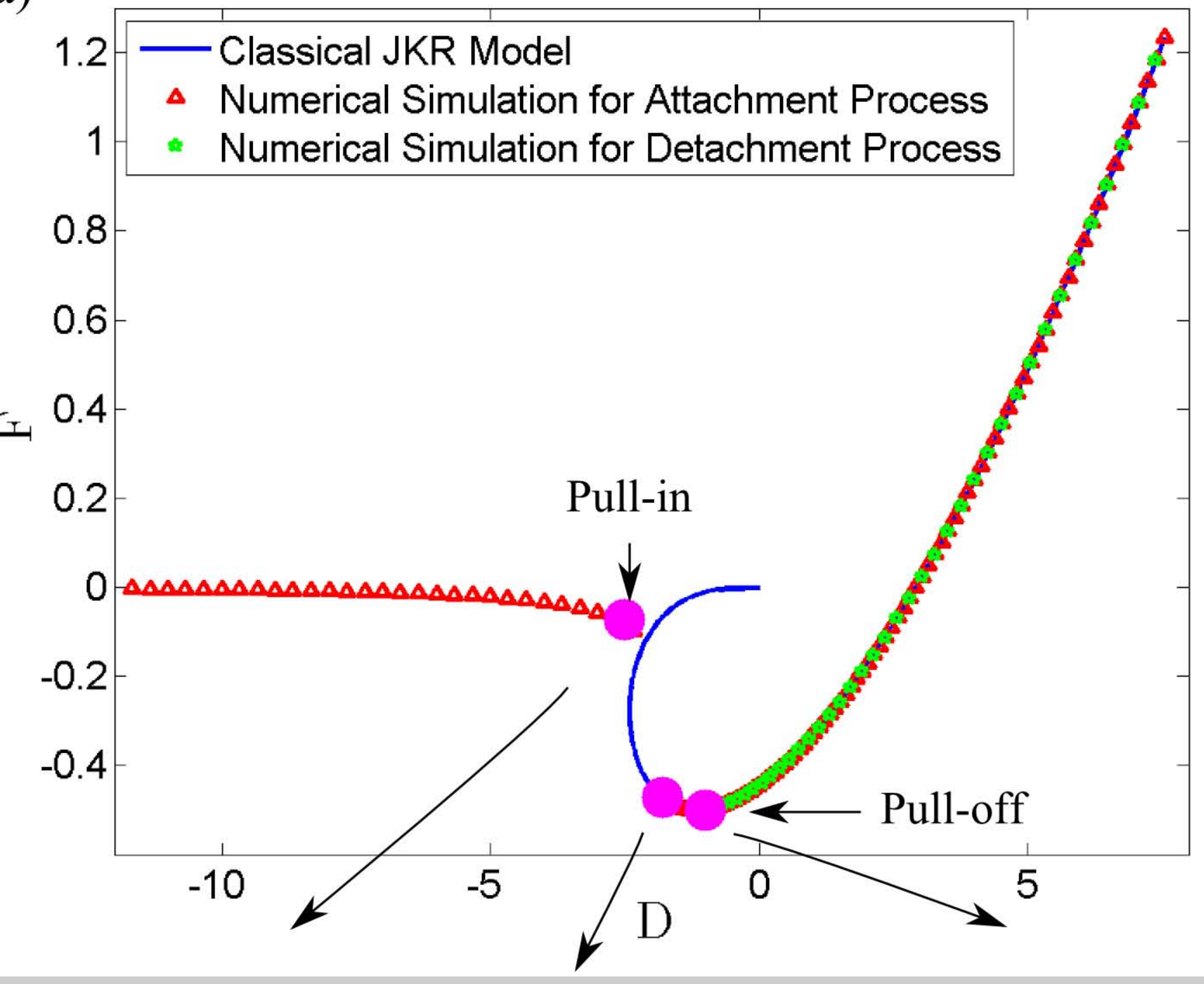

(b)

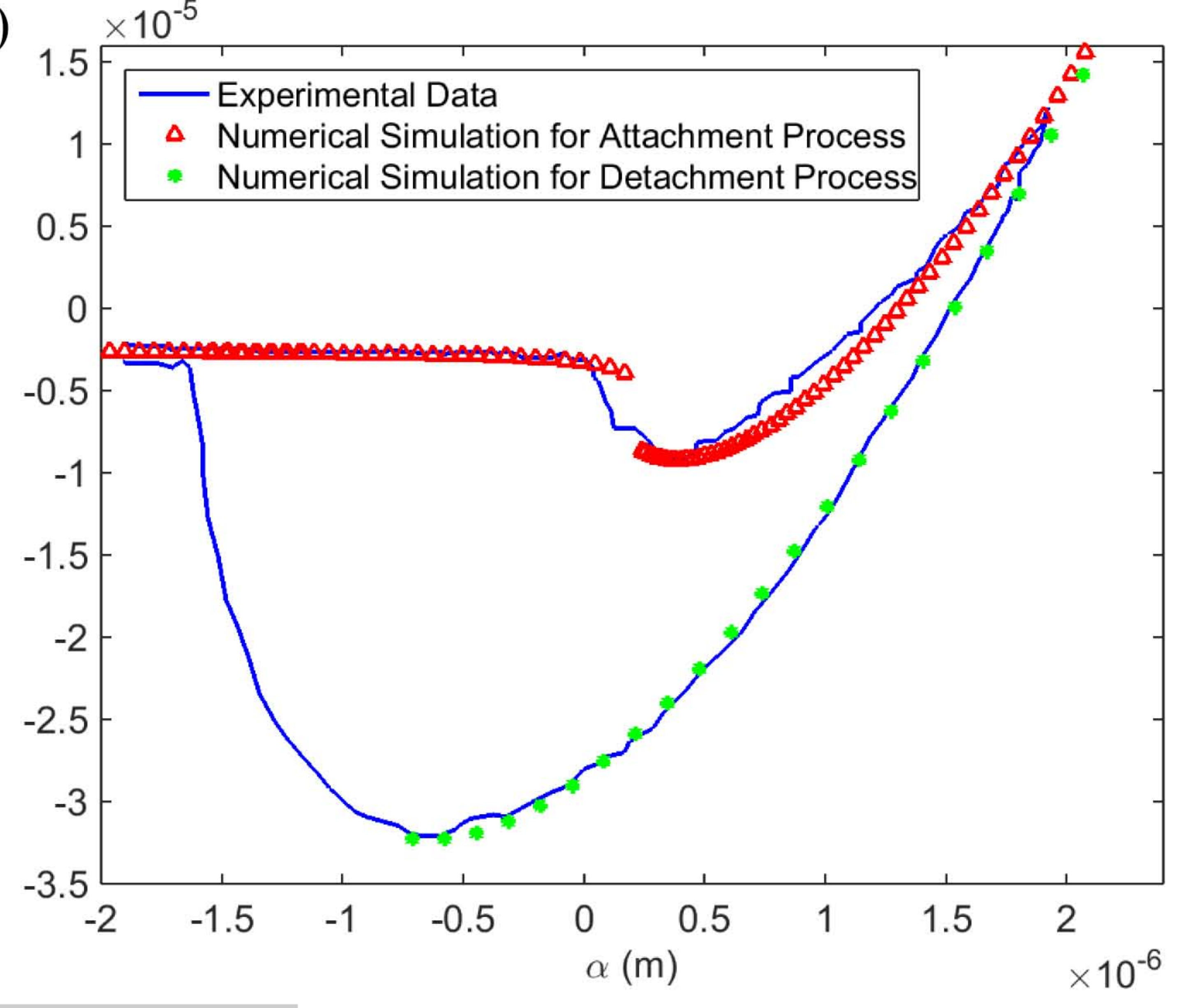

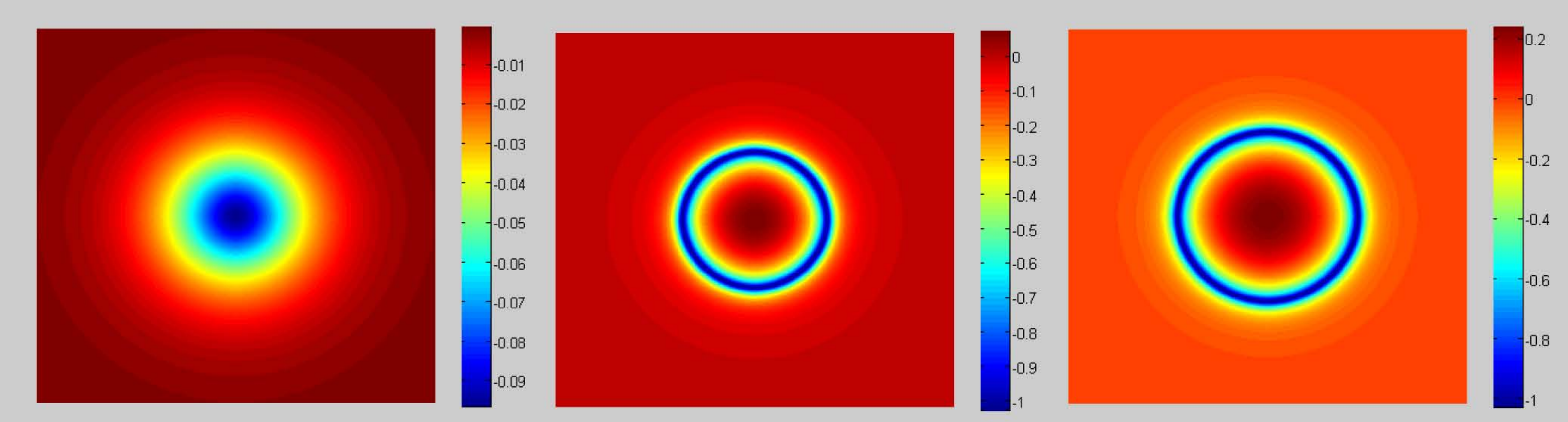


(a)

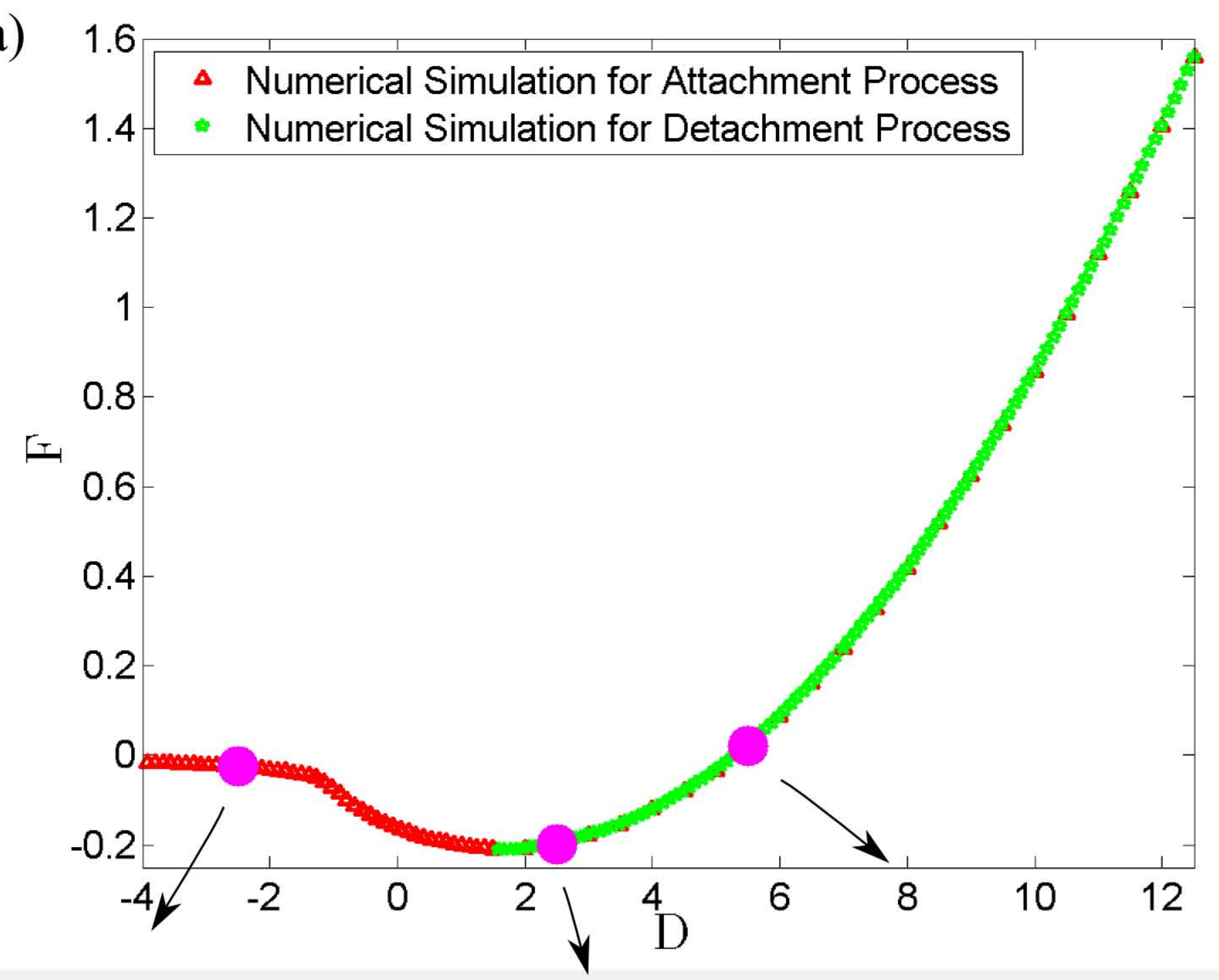

(b)

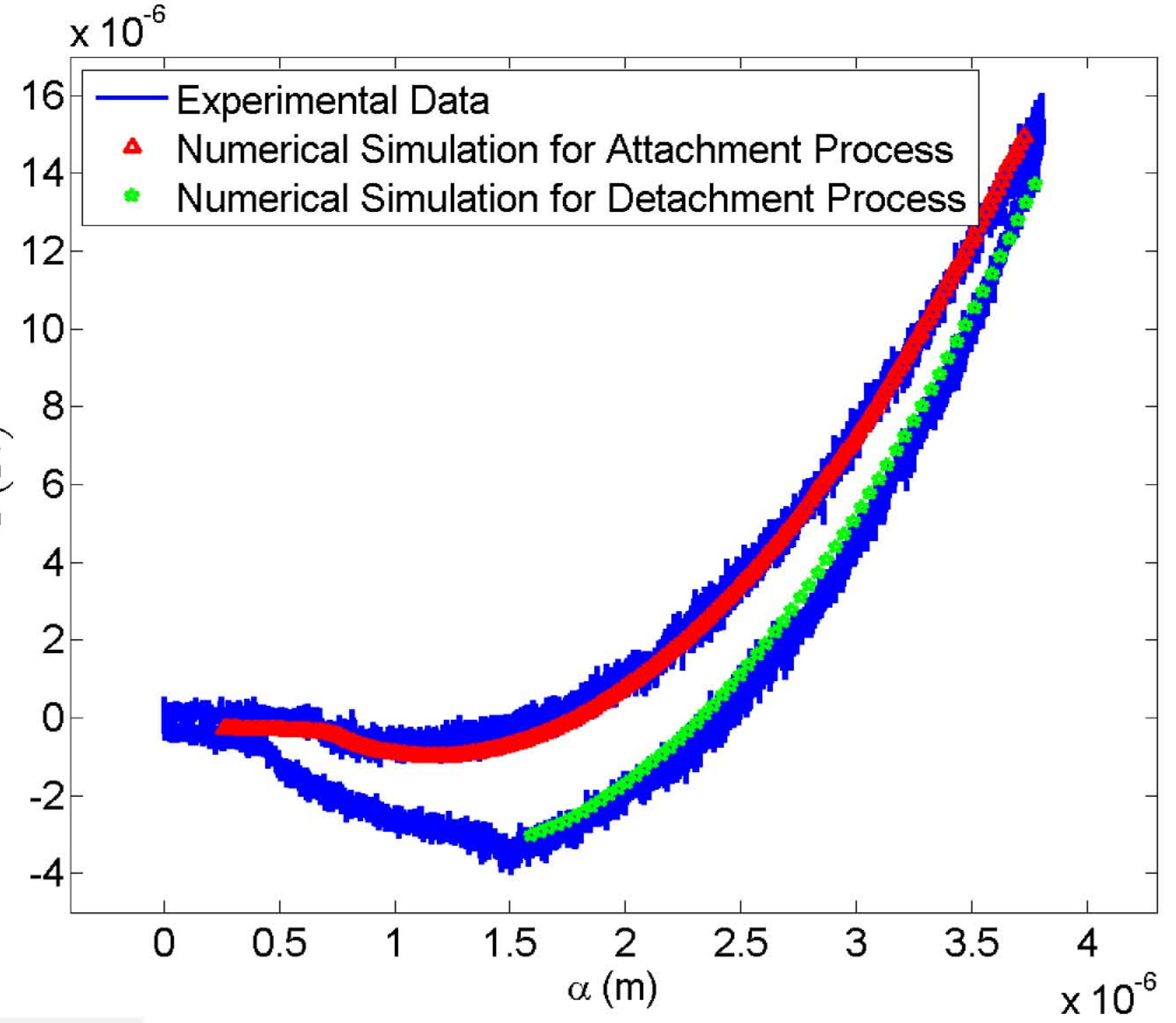


(a) $\quad 4 \times 10^{-5}$

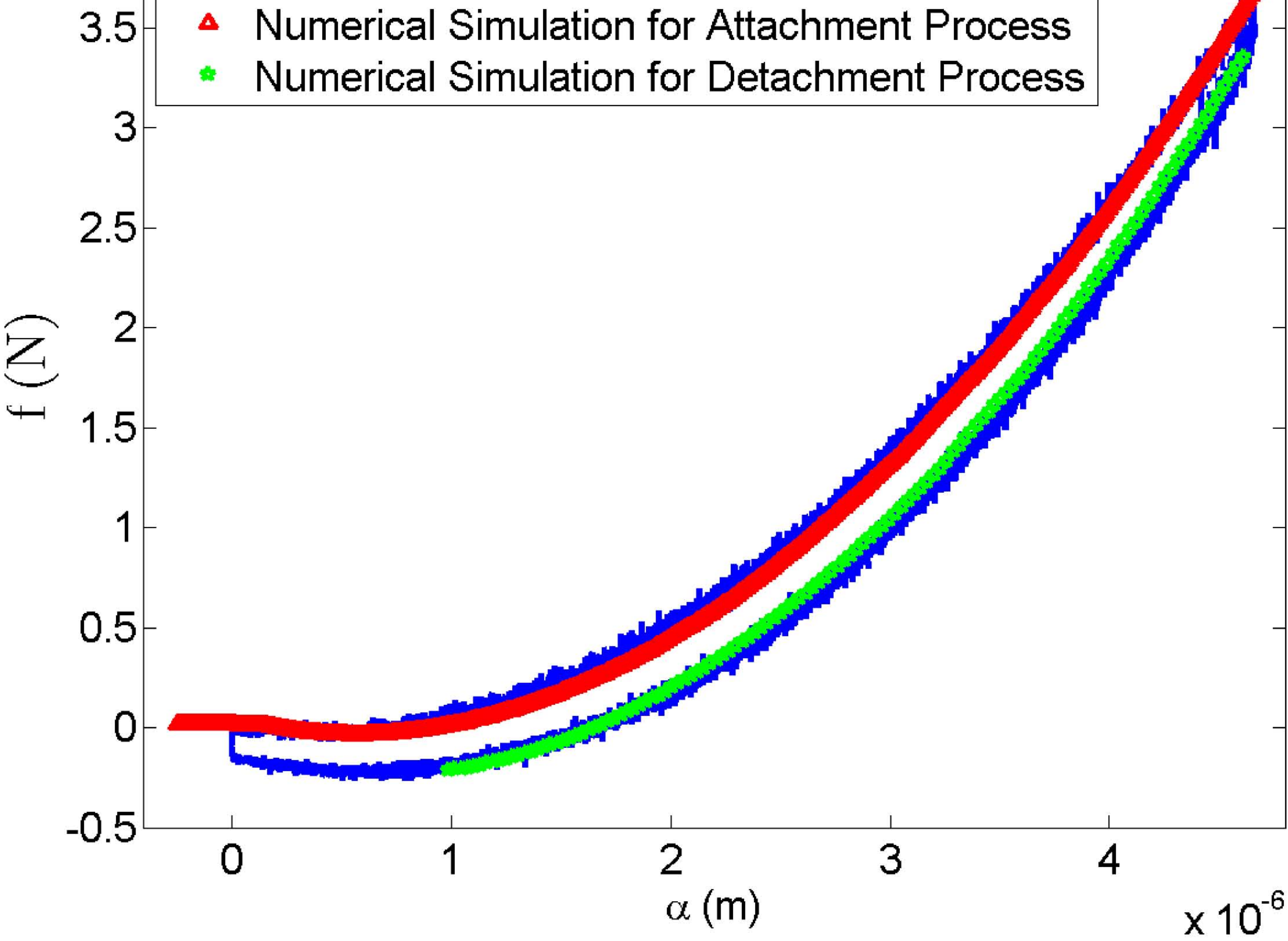

(b) $20 \times 10^{-6}$

$\Delta$ Numerical Simulation for Attachment Process

15

- Numerical Simulation for Detachment Process

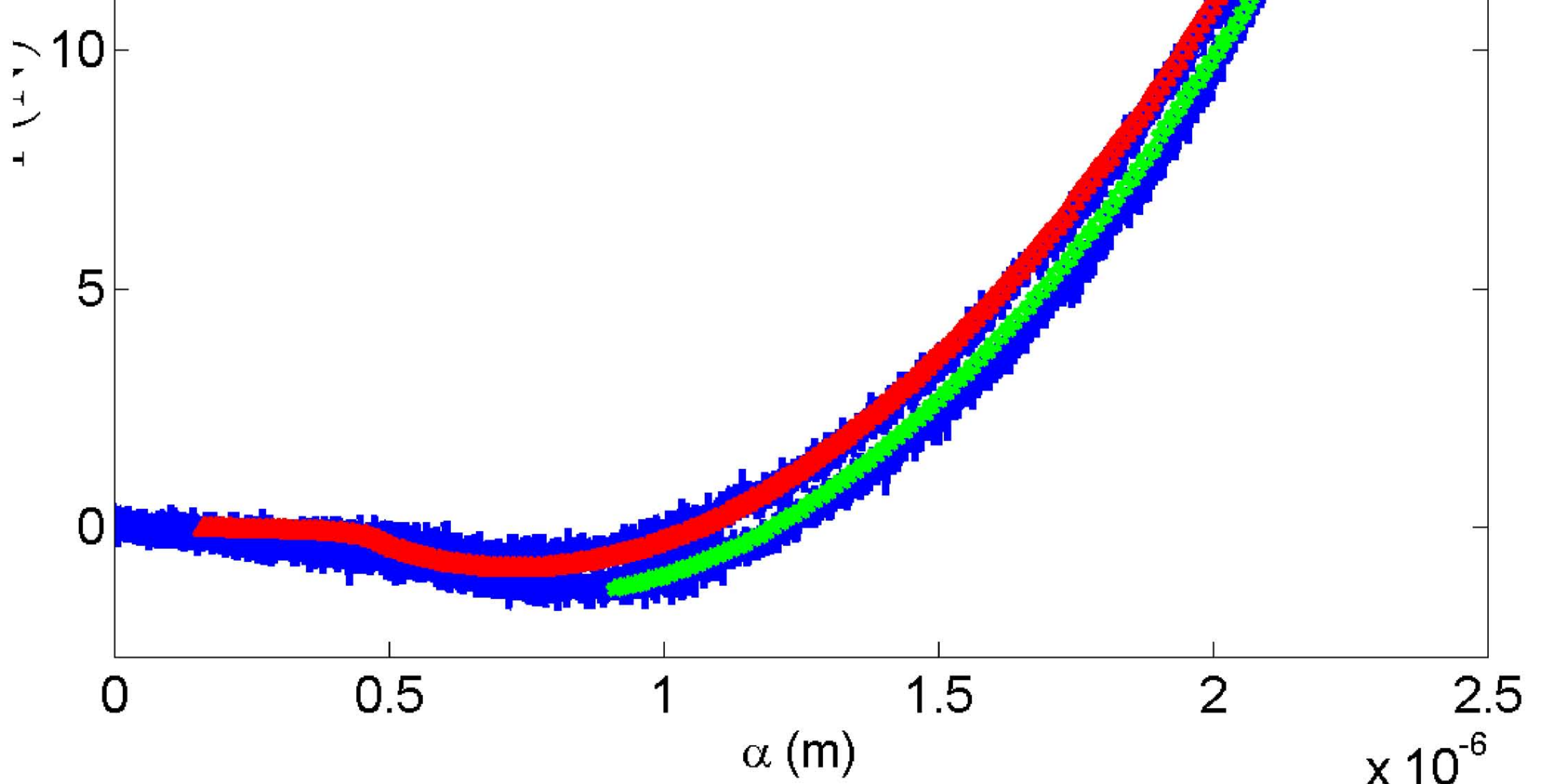


(a)

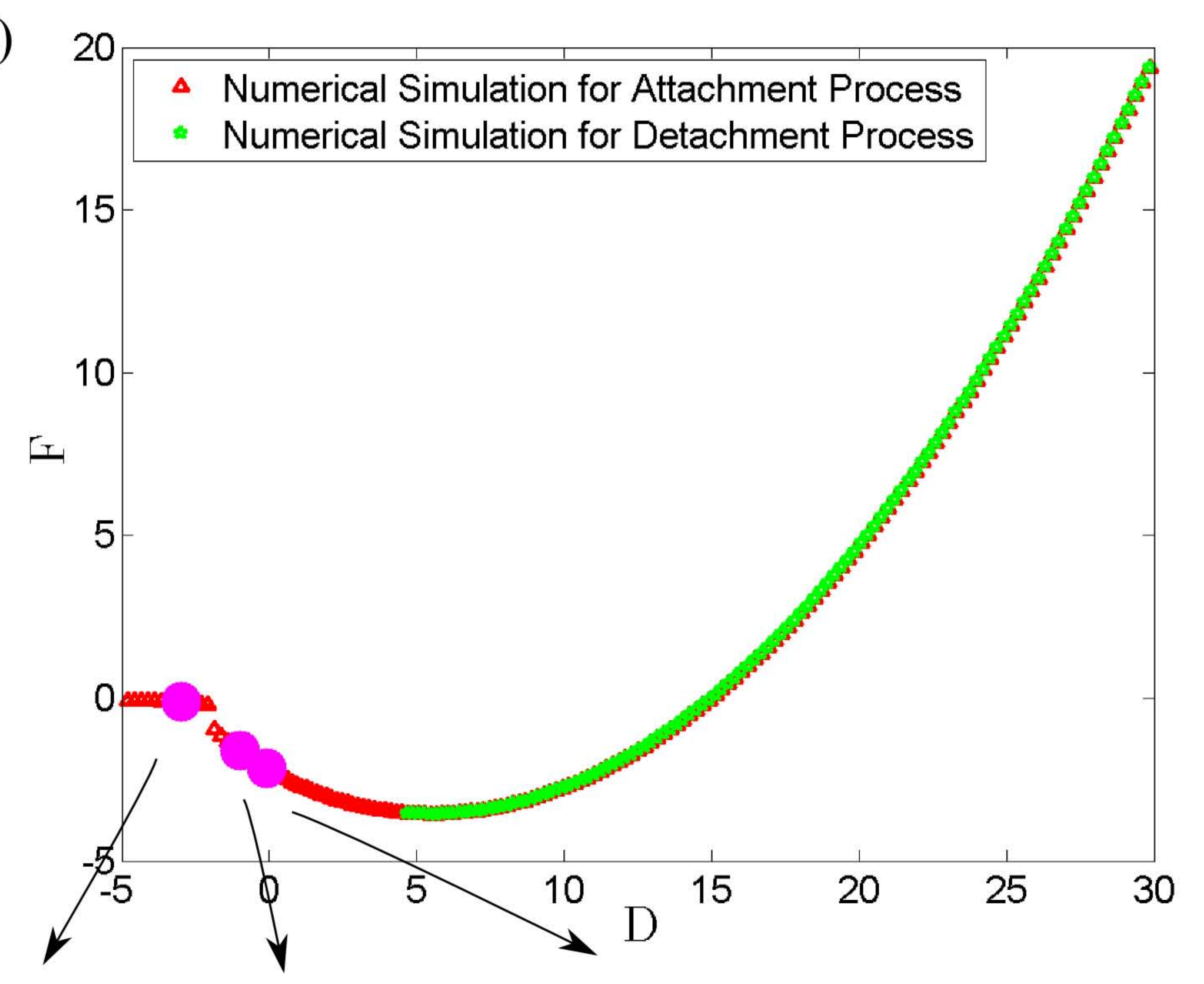

(b)

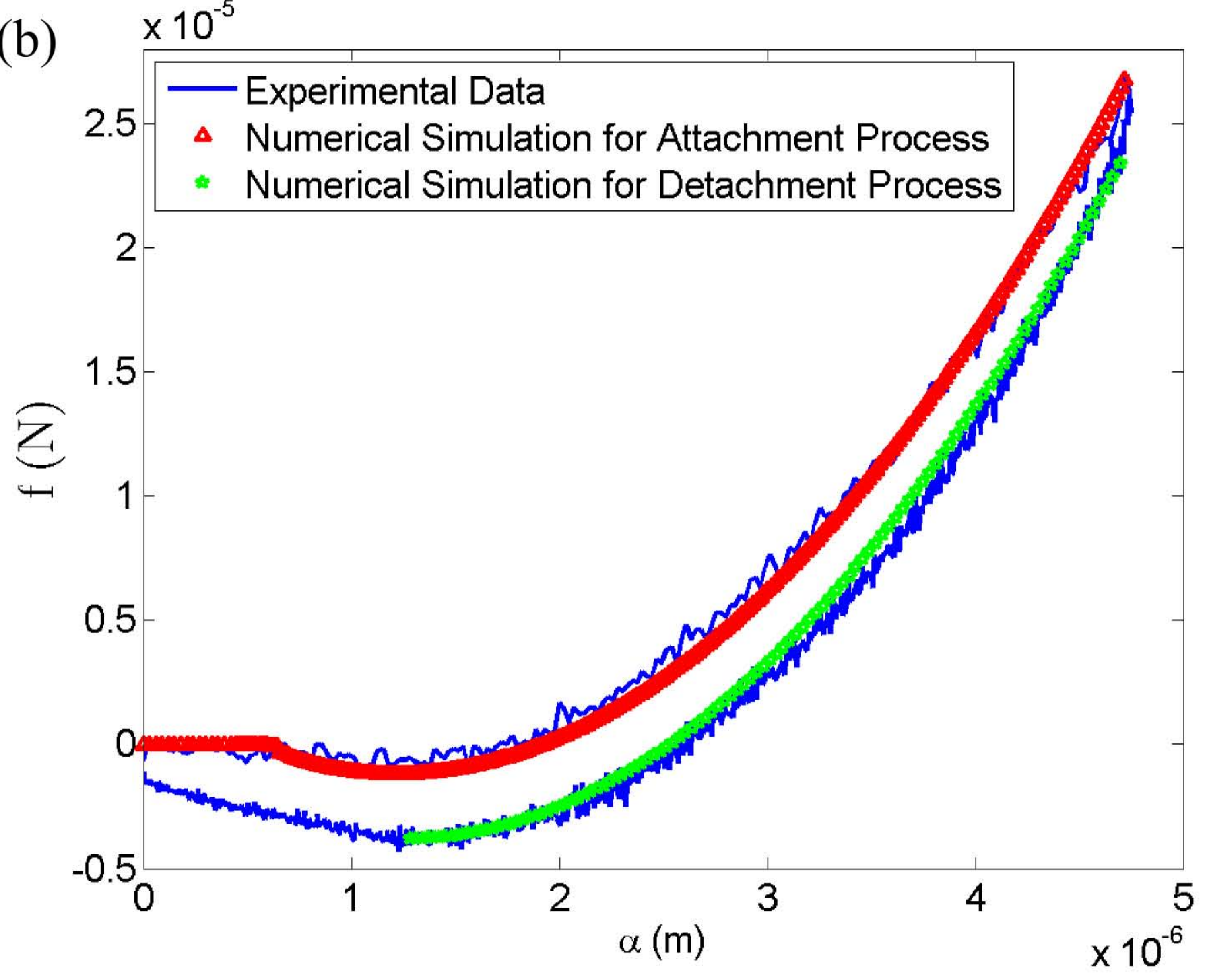




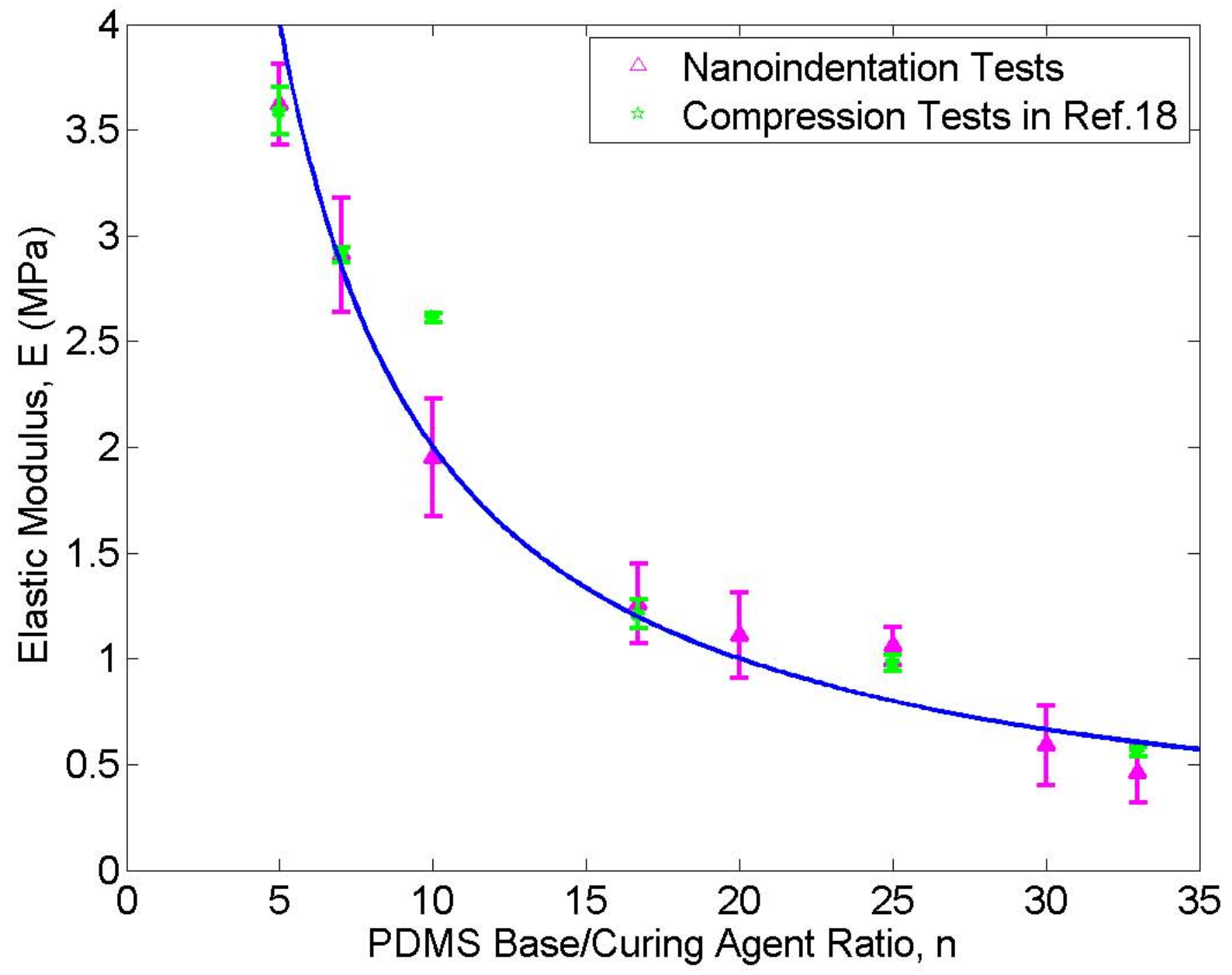

